\title{
EP-110
}

\section{Gallbladder clear cell carcinoma: A case report}

\author{
Bayanduuren BATSUURI*
}

General Surgery Department, Grandmed Hospital, Ulaanbaatar, Mongolia

Introduction: Worldwide, the highest incidence rates (up to 7.5 per 100,000 in male and 23 per 100,000 in female) occur among populations in the Western part of South America (Chile and Peru), in North American Indians, in Mexican Americans, and in Northern India. The best characterized risk factor for the development of gallbladder cancer is chronic inflammation associated with gallstones. Metastatic clear cell carcinoma in the gallbladder is extremely rare, with reported frequencies of less than $0.6 \%$ in large autopsy reviews.

Methods: Patient was a 54 year old female. Patient was right upper abdominal pain and epigastric middle pain. Nausea and vomiting, pain was started 10 days ago, after ate dumplings. No history of previous any gastrointestinal disease. The physical examination: Tenderness in right hypochondrium. Lump in the hypochondrium. Murphy's sign was positive. The patient's in blood test increased white blood cell and C-reactive protein. Abdominal ultrasound examination: Gallbladder is enlargement, about $6.0 \mathrm{~cm} \times 3.0 \mathrm{~cm}$ like the mudstone in the gallbladder. GB wall thickening/double, common bile duct is dilated $1.2 \mathrm{~cm}$. Abdominal contrast computed tomography examination: Moderate thickening with surrounding mild edematous changes in wall of the gallbladder. Size of the GB is moderated dilated with diffuse.

Results: We were emergency surgery of under general anesthesia open cholecystectomy and intraoperative cholangiogram. During surgery gallbladder was distended and gallbladder wall very thickness, hardness. In cholangiogram common bile duct no obstructive sign.

Conclusions: Clear cell carcinoma very rare disease. Our team diagnosed during surgery and histological analysis. 